\title{
A quantum algorithm that deletes marked states from an arbitrary database
}

\author{
LIU Yang ${ }^{1,2^{*}} \&$ OUYANG XiaoPing ${ }^{3}$ \\ ${ }^{1}$ School of Nuclear Science and Engineering, North China Electric Power University, Beijing 102206, China; \\ ${ }^{2}$ Department of Physics, Tsinghua University, Beijing 100084, China; \\ ${ }^{3}$ Northwest Institute of Nuclear Technology, Xi'an 710024, China
}

Received December 23, 2012; accepted February 24, 2013

We present a general quantum deletion algorithm that deletes $M$ marked states from an $N$-item quantum database with arbitrary initial distribution. The general behavior of this algorithm is analyzed, and analytic result is given. When the number of marked states is no more than $\frac{3 N}{4}$, this algorithm requires just a single query, and this achieves exponential speedup over classical algorithm.

quantum deletion algorithm, quantum search algorithm, Grover algorithm, Long algorithm

Citation: Liu Y, Ouyang X P. A quantum algorithm that deletes marked states from an arbitrary database. Chin Sci Bull, 2013, 58: 2329-2333, doi: 10.1007/s11434-0135885-0

Developments of quantum information processing have been fruitful in recent years. A quantum computer can solve tasks which are hard for classical computer [1-5]. For example, a quantum computer can determine a given function that is constant or balanced with a single evaluation which is exponential speeding over its classical algorithm [1]. A quantum large integer factoring algorithm [2] and Grover search algorithm [3] can complete tasks exponentially faster than classical counterparts. Quantum algorithms have attracted much attention and been further developed and applied to various problems [6-10], recent interests have been focused on quantum algorithms using duality mode in a quantum register $[11,12]$.

Deleting an item from a database is a routine task in database processing. In classical computing, deleting operation is an essential method to preserve data structure for convenient searching and visiting. For instance, in the basis technique of well-known Google search engine - PageRank algorithm $[13,14]$, the most frequently clicked $N$ items are selected from the magnanimity of information. Then the first $N$ nodes form a heap structure and web pages with respect to the marked nodes are deleted to preserve the first $N$ nodes

*Corresponding author (email: yliu@ncepu.edu.cn) dynamically. Generally, classical deleting is considered to be equivalent to classical searching. To delete $M$ marked items from a $N$-item database usually requires $O(M N)$ steps. At present, Fibonacci heaps algorithm [15] as the optimal classical deletion algorithm [16] can delete $M$ marked items from an $N$-item heap with total complexity of $O(N \log N+M)$.

Deleting items in a database is a widely met scientific problem in quantum computing. Some certain items do not satisfy the computing demands, then one should delete them. We have proposed quantum deletion algorithm [17] that deletes a marked item from unsorted $N=2^{n}$ item database. However, in a variety of practical cases, it would be desirable to apply it to deleting multiple number of marked items and non-uniform initial distribution, where $N$ is not essentially the power of 2 . This could arise in situations where the deletion is used as a subroutine in a large quantum computation. Another example would be the given that initial distribution is intrinsically non-uniform and the marked solution is not unique.

In this article, we generalize a quantum deletion algorithm [17] to the case that the number of marked items is more than one, the initial amplitudes are complex and follow an arbitrary distribution. Moreover we analyze the general behavior of deletion operator and give analytic results. Finally, we 
study three cases in which only single query is required for deleting.

\section{Generalized deletion algorithm on quantum computer}

The abstract problem we consider is the most general case: if there is a quantum database with $N$ items, where $N$ is an arbitrary integer. The initial amplitude of basis items are arbitrary complex numbers. $M$ items $\tau_{1}, \tau_{2}, \cdots \tau_{M}$ satisfy a query function $f(\tau)=1$, and other items satisfy $f(x)=0$, the task is deleting $M$ items $\tau_{1}, \tau_{2}, \cdots \tau_{M}$ from $N$-item quantum database with $100 \%$ success rate. The initial state of the quantum database is prepared in $\left|\psi_{0}\right\rangle$,

$$
\left|\psi_{0}\right\rangle=|\gamma\rangle=a_{0}|0\rangle+a_{1}|1\rangle+\cdots+a_{N-1}|N-1\rangle,
$$

where $|c\rangle$ is the normalized state sum over all non-marked states, and $|\tau\rangle$ is the normalized state sum over all $M$ marked states,

$$
\begin{aligned}
& |c\rangle=\sqrt{\frac{1}{\sum_{i \neq \tau}\left|a_{i}\right|^{2}}} \sum_{i \neq \tau} a_{i}|i\rangle, \\
& |\tau\rangle=\sqrt{\frac{1}{\sum_{i=\tau_{1}}^{\tau_{M}}\left|a_{i}\right|^{2}}}\left(a_{\tau_{1}}\left|\tau_{1}\right\rangle+a_{\tau_{2}}\left|\tau_{2}\right\rangle+\cdots+a_{\tau_{M}}\left|\tau_{M}\right\rangle\right) .
\end{aligned}
$$

Then we can express $\left|\psi_{0}\right\rangle$ in the two-dimensional space spanned by $|c\rangle$ and $|\tau\rangle$,

$$
\left|\psi_{0}\right\rangle=|\gamma\rangle=U|0\rangle=\cos \beta|c\rangle+\sin \beta|\tau\rangle,
$$

where the normalized coefficients

$$
\begin{aligned}
& \cos \beta=\sqrt{\sum_{i \neq \tau}\left|a_{i}\right|^{2}}=\sqrt{\sum_{i \neq \tau}|\langle i|U| 0\rangle|^{2}}, \\
& \sin \beta=\sqrt{\sum_{i=\tau_{1}}^{\tau_{M}}\left|a_{i}\right|^{2}}=\sqrt{\sum_{i=\tau_{1}}^{\tau_{M}}|\langle i|U| 0\rangle|^{2}} .
\end{aligned}
$$

The unitary operator $U$ can transform $|00 \cdots 0\rangle$ into $|\gamma\rangle$ state. When $|\gamma\rangle$ is known, $U$ operation can be constructed from a scheme proposed in [18]. For example, if a database with only two items in the form of 4-qubit GHZ state, $|\gamma\rangle=$ $\frac{1}{\sqrt{2}}(|0000\rangle+|1111\rangle)$, the $U$ operation is illustrated in Figure 1 .

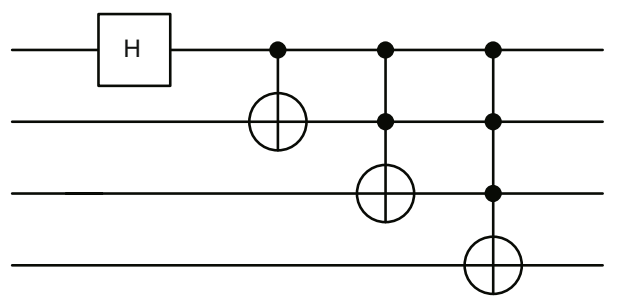

Figure 1 Quantum circuit for implementing the 4-qubit GHZ state from $|0000\rangle$ state. $\mathrm{H}$ represents the Hadamard-Walsh transformation and $\bigoplus$ represents the NOT gate.
The generalized quantum deletion algorithm consists of successive applications of a quantum deletion subroutine, indicated as $S$ operation. The $S$ operation consists of four steps:

Step 1: Except the $M$ marked states from $\left|\tau_{1}\right\rangle$ to $\left|\tau_{M}\right\rangle$, perform a conditional phase shift $e^{i \phi}$ to all non-marked computational basis state, this step can be denoted as $I_{c}$,

$$
I_{c}=I+\left(e^{i \phi}-1\right) \sum_{i \neq \tau}|i\rangle\langle i|,
$$

where $\phi$ will be given later.

Step 2: Perform a $n$-qubit operation $U^{\dagger}$, where $U$ transform $n$-qubit $|0\rangle$ state to $|\gamma\rangle$ state.

Step 3: Perform a conditional phase shift $-e^{i \phi}$ to $|0\rangle$ state and perform $e^{i \pi}$ to all other basis states. This step can be denoted as $-I_{0}$,

$$
-I_{0}=-I-\left(e^{i \phi}-1\right)|0\rangle\langle 0| .
$$

Step 4: Perform the $n$-qubit transformation $U$.

In the space spanned by $|c\rangle$ and $|\tau\rangle$, above deletion operator subroutine $S$ can be expressed by a matrix

$$
\begin{aligned}
S & =-U I_{0} U^{\dagger} I_{c} \\
& =\left[\begin{array}{cc}
-e^{i \phi}\left(1+\left(e^{i \phi}-1\right) \cos ^{2} \beta\right) & -\left(e^{i \phi}-1\right) \sin \beta \cos \beta \\
-e^{i \phi}\left(e^{i \phi}-1\right) \sin \beta \cos \beta & -e^{i \phi}+\left(e^{i \phi}-1\right) \cos ^{2} \beta
\end{array}\right] .
\end{aligned}
$$

In the above procedures, the phase matching condition in quantum search algorithm [19-24] is required where the two phases are equal. Suppose the $M$ marked items can be deleted in $J$ iterations with $100 \%$ success rate, we explicitly work out the phase $\phi$ using the $S O(3)$ picture of quantum algorithm [22,23],

$$
\phi=2 \arcsin \left(\frac{\sin \frac{\pi}{4 J+2}}{\cos \beta}\right) .
$$

Eq. (8) has real solutions for

$$
J \geqslant \frac{\pi}{2 \pi-4 \beta}-\frac{1}{2}
$$

otherwise there will be no real solution. The optimal iteration number is

$$
J_{\mathrm{op}}= \begin{cases}j_{m} & \text { if } j_{m} \text { is an integer, } \\ \mathrm{INT}\left[j_{m}\right]+1 & \text { if } j_{m} \text { is not an integer }\end{cases}
$$

where

$$
j_{m}=\frac{\pi}{2 \pi-4 \beta}-\frac{1}{2}
$$

and INT[ ] means taking the integer part. In our deletion algorithm, an integer $J \geqslant J_{\text {op }}$ fixes a phase rotation that deletes the $M$ marked states with certainty in $J$ iterations. For convenience, $J$ is usually chosen to be the lower bound $J=J_{\text {op }}$ in most cases. The optimal iteration number $J_{\text {op }}$ versus the database parameter $\beta$ is given in Figure 2. When the number of marked states is small, the number of queries is very small. 


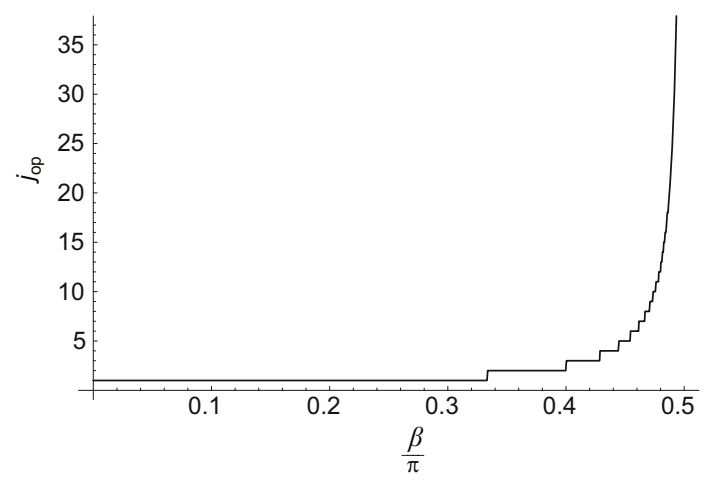

Figure 2 The optimal iteration number $j_{\mathrm{op}}$ versus $\beta$.

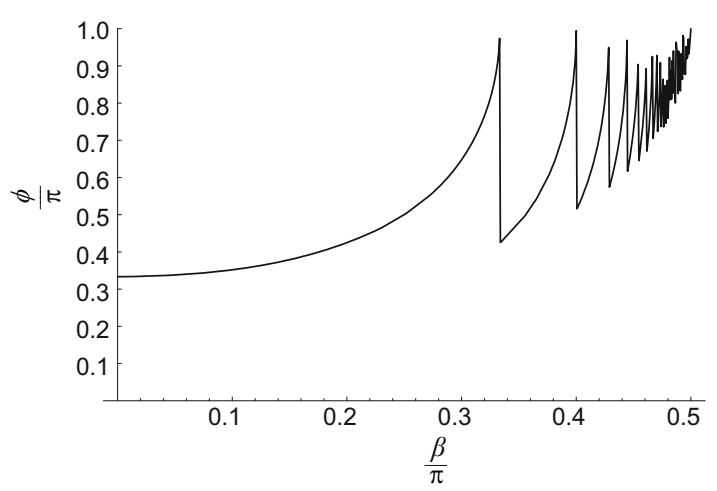

Figure 3 The phase rotation $\phi$ versus $\beta$ for $J=J_{\mathrm{op}}$.

When $J=J_{\text {op }}, \beta$ fixes a phase rotation $\phi$ which is shown in Figure 3.

Usually we choose $J$ to be $J_{\text {op }}$ for a given $\beta$, which in turn depends on the normalized coefficient in the initial state of quantum database. Then $M$ marked state will be deleted with $100 \%$ success rate in $J$ iterations. From Figures 2 and 3, we can see that if the proportion of marked states $\sin ^{2} \beta$ does not exceed $\frac{3}{4}$, i.e. $0<\beta \leqslant \frac{\pi}{3}$, then $J=1$ and $\phi=2 \arcsin \left(\frac{1}{2 \cos \beta}\right)$, this algorithm only requires a single query in deleting processing. The case with an even superposition of $N=2^{n}$ basis states and a unique marked state is a special case of this general scenario [17], where $\sin \beta=\sqrt{\frac{1}{N}}, \phi=2 \arcsin \left(\frac{1}{2} \sqrt{\frac{N}{N-1}}\right)$ and $J=1$. For $\frac{\pi}{3}<\beta \leqslant \frac{2 \pi}{5}$, we calculate out that $J=2$ and $\phi=2 \arcsin \left(\frac{\sin \frac{\pi}{10}}{\cos \beta}\right)$. For $\frac{2 \pi}{5}<\beta \leqslant \frac{3 \pi}{7}$, we calculate out that $J=3$ and $\phi=2 \arcsin \left(\frac{\sin \frac{\pi}{14}}{\cos \beta}\right)$, and so on.

\section{Properties of generalized quantum deletion algorithm}

In the following part of this article, we focus on the performance of arbitrary $k$ iterations of $S$. We can find that the deletion algorithm is effective for certain times of iteration. We rewrite the expression of $S$ in a diagonalized form

$$
S=T \Lambda T^{\dagger},
$$

where $T$ is

$$
\begin{aligned}
\sqrt{\frac{1}{R}}\left[\begin{array}{cc}
e^{-\frac{i \phi}{2}}\left(\cos \frac{\phi}{2} \cos \beta+\cos \beta^{\prime}\right) & -\sin \beta \\
\sin \beta & e^{\frac{i \phi}{2}}\left(\cos \frac{\phi}{2} \cos \beta+\cos \beta^{\prime}\right)
\end{array}\right] \\
\Lambda=\left[\begin{array}{cc}
-e^{i\left(\phi+2 \beta^{\prime}\right)} & 0 \\
0 & -e^{i\left(\phi-2 \beta^{\prime}\right)}
\end{array}\right] \\
\beta^{\prime}=\arcsin \left(\sin \frac{\phi}{2} \cos \beta\right) \\
R=\sin ^{2} \beta+\left(\cos \frac{\phi}{2} \cos \beta+\cos \beta^{\prime}\right)
\end{aligned}
$$

Using eq. (4) and suppose that

$$
\sin \left(\frac{\pi}{4 J+2}\right)=q, \quad \frac{\phi}{2}=\arcsin \left(\frac{q}{\cos \beta}\right),
$$

we obtain

$$
\begin{aligned}
& \sin \frac{\phi}{2}=\frac{q}{\cos \beta}, \quad \cos \frac{\phi}{2}=\frac{\sqrt{\cos ^{2} \beta-q^{2}}}{\cos \beta}, \\
& \beta^{\prime}=\arcsin q, \quad R=2-2 q^{2}+2 \sqrt{\left(1-q^{2}\right)\left(\cos ^{2} \beta-q^{2}\right)} .
\end{aligned}
$$

Successive $k$ times of $S$ iteration can be analytically written as

$$
S^{k}=T S^{k} T^{\dagger}=(-1)^{k} e^{i k \phi}\left[\begin{array}{cc}
\cos \theta+i \sin \theta \sqrt{\frac{\cos ^{2} \beta-q^{2}}{1-q^{2}}} & \sin \theta \tan \beta \sqrt{\frac{q^{2}}{1-q^{2}}}+i \sin \theta \tan \beta \sqrt{\frac{\cos ^{2} \beta-q^{2}}{1-q^{2}}} \\
-\sin \theta \tan \beta \sqrt{\frac{q^{2}}{1-q^{2}}}+i \sin \theta \tan \beta \sqrt{\frac{\cos ^{2} \beta-q^{2}}{1-q^{2}}} & \cos \theta-i \sin \theta \sqrt{\frac{\cos ^{2} \beta-q^{2}}{1-q^{2}}}
\end{array}\right]
$$

and

$$
\theta=2 k \beta^{\prime}=2 k \arcsin q=\frac{k \pi}{2 J+1} .
$$

We analyze the general properties of our algorithm.

(i) We now analyze the periodic property of our algo- rithm. Functions such as $\sin \theta$ and $\cos \theta$ vary periodically with a period of $4 J+2$ in $k$, and functions of $(-1)^{k} \sin \theta$ and $(-1)^{k} \cos \theta$ vary periodically with a period $2 J+1$ in $k$. Hence expect the global phase rotation $e^{i k \phi}, S^{k}$ is a periodic function of $k$ with a period $2 J+1$. 
(ii) Successive $k$ iterations of $S$ make the initial state $|\gamma\rangle$ become $\left|\psi_{k}\right\rangle$,

$$
e^{i k(\phi+\pi)}\left[\begin{array}{c}
\cos \theta \cos \beta+\frac{\sin \theta \sin ^{2} \beta}{\cos \beta} \sqrt{\frac{q^{2}}{1-q^{2}}}+i \frac{\sin \theta}{\cos \beta} \sqrt{\frac{\cos ^{2} \beta-q^{2}}{1-q^{2}}} \\
-\sin \theta \sin \beta \sqrt{\frac{q^{2}}{1-q^{2}}}+\cos \theta \cos \beta
\end{array}\right] .
$$

We can find that if and only if $\tan \frac{k \pi}{2 J+1}=\left|\cot \frac{\pi}{4 J+2}\right|$, i.e. $k=J+(2 J+1) n$, where $n$ is arbitrary nonnegative number, the amplitude of the marked state $|\tau\rangle$ is zero. Thus $J+(2 J+1) n$ times of $S$ iteration construct an effective deletion algorithm. For $n=0$, it reduces to the algorithm proposed in Section 1 . After $J+(2 J+1) n$ iterations, we calculate out the final state from eq. (20)

$$
\left|\psi_{k}\right\rangle=e^{i\left[\left(J+\frac{1}{2}\right) \pi+\left(k-\frac{1}{2}\right) \phi\right]}|c\rangle .
$$

This type of iteration processing can be represented as

$$
|\gamma\rangle \stackrel{S^{J+(2 J+1) n}}{\longrightarrow} e^{i\left[\left(J+\frac{1}{2}\right) \pi+\left(k-\frac{1}{2}\right) \phi\right]}|c\rangle .
$$

(iii) Usually, the proportion of the marked states among the initial state is not very large, so $\sum_{i=\tau_{1}}^{\tau_{M}}\left|a_{i}\right|^{2} \leqslant \frac{3}{4}$, i.e. $\beta \leqslant \frac{\pi}{3}$ is satisfied in generic initial conditions, for example the problem in [17]. Then a single query is required in this deletion algorithm. Accordingly, $S^{k}$ operation becomes an effective deletion processing with a period 3 in iteration number. We look at three cases for quantum database in which $\beta \leqslant \frac{\pi}{3}$.

Case 1: When $k=3 n+1$, i.e. $k=1,4,7,10,13 \cdots, S^{k}$ in eq. (18) is reduced to the following form:

$$
e^{i k \phi}\left[\begin{array}{cc}
-\frac{1}{2}-i \sqrt{\cos ^{2} \beta-\frac{1}{4}} & -\frac{\tan \beta}{2}-i \tan \beta \sqrt{\cos ^{2} \beta-\frac{1}{4}} \\
\frac{\tan \beta}{2}-i \tan \beta \sqrt{\cos ^{2} \beta-\frac{1}{4}} & -\frac{1}{2}+i \sqrt{\cos ^{2} \beta-\frac{1}{4}}
\end{array}\right] .
$$

This type of transformation can be represented as

$$
|\gamma\rangle \underset{\beta \leqslant \frac{\pi}{3}}{\stackrel{S^{3 n+1}}{\longrightarrow}} e^{i\left[\left(k-\frac{\phi}{2}\right) \phi-\frac{1}{2}\right]}|c\rangle .
$$

After $k=3 n+1$ times of deletion iteration, the $M$ marked states will be successfully deleted from the database for $\beta \leqslant$ $\frac{\pi}{3}$. The global phase factor can be left alone, or be eliminated by a simultaneous phase rotation of $e^{-i\left[\left(k-\frac{\phi}{2}\right) \phi-\frac{1}{2}\right]}$ to all basis states. Consider a particular problem which deletes one marked item from an unsorted database [17], eq. (24) is reduced to following result that results in [17]:

$$
\sqrt{\frac{1}{N}} \sum_{i=0}^{N-1}|i\rangle \underset{\beta=\arcsin \sqrt{\frac{1}{N}}}{\stackrel{S^{3 n+1}}{\longrightarrow}} e^{i\left[\left(k-\frac{\phi}{2}\right) \phi-\frac{1}{2}\right]} \sqrt{\frac{1}{N-1}} \sum_{i \neq \tau}|i\rangle .
$$

Case 2: When $k=3 n+2$, i.e. $k=2,5,8,11,14 \cdots, S^{k}$ in eq. (18) can be rewritten as

$$
e^{i k \phi}\left[\begin{array}{cc}
-\frac{1}{2}+i \sqrt{\cos ^{2} \beta-\frac{1}{4}} & \frac{\tan \beta}{2}+i \tan \beta \sqrt{\cos ^{2} \beta-\frac{1}{4}} \\
-\frac{\tan \beta}{2}+i \tan \beta \sqrt{\cos ^{2} \beta-\frac{1}{4}} & -\frac{1}{2}-i \sqrt{\cos ^{2} \beta-\frac{1}{4}}
\end{array}\right] .
$$

This type of transformation can be represented as

$$
|\gamma\rangle \underset{\beta \leqslant \frac{\pi}{3}}{\stackrel{S^{3 n+2}}{\longrightarrow}} e^{i[(k+1) \phi+\pi]} \cos \beta|c\rangle+e^{i(k \phi+\pi)} \sin \beta|\tau\rangle .
$$

$k=3 n+2$ times of iteration cannot delete the marked states, and it only adds different phases to $|c\rangle$ and $|\tau\rangle$. For deleting one marked state from unsorted database, eq. (27) can be deduced to the following result in [17]:

$$
\sqrt{\frac{1}{N}} \sum_{i=0}^{N-1}|i\rangle \underset{\beta=\arcsin \sqrt{\frac{1}{N}}}{\stackrel{S^{3 n+2}}{\longrightarrow}} \frac{e^{i[(k+1) \phi+\pi]}}{\sqrt{N}} \sum_{i \neq \tau}|i\rangle+\frac{e^{i(k \phi+\pi)}}{\sqrt{N}}|\tau\rangle .
$$

Case 3: When $k=3 n+3$, i.e. $k=3,6,9,12,15 \cdots$, we may reduce $S^{k}$ in eq. (18) to $e^{i k \phi} I$. This type of iterations can be represented as

$$
|\gamma\rangle \underset{\beta \leqslant \frac{\pi}{3}}{\stackrel{S^{3 n+3}}{\longrightarrow}} e^{i k \phi}|\gamma\rangle .
$$

So except a global irrelevant total phase $e^{i k \phi}, k=3 m+3$ times of iteration leave the state of the system still in the initial state. This type of operation does not delete any thing, which coincides to result in [17]:

$$
\sqrt{\frac{1}{N}} \sum_{i=0}^{N-1}|i\rangle \stackrel{S^{3 m+3}}{\longrightarrow} e^{i k \phi} \sqrt{\frac{1}{N}} \sum_{i=0}^{N-1}|i\rangle .
$$

\section{Conclusions}

In summary, a generalized quantum deletion algorithm with certainty is presented. This algorithm deletes $M$ marked states from arbitrary $N$-item initial quantum database in $J$ iterations. The deletion operation is periodic with a period $2 J+1$. For quantum database where $\beta \leqslant \frac{3}{4}$, this algorithm requires a single query which achieves an exponential speedup over classical computation. This generalized deletion algorithm uses an arbitrary quantum database, which is also used in quantum amplitude amplification (QAA) [25]. Different from QAA where the task is to find out the marked states, here we delete the marked states from the superposition. If we exchange the role of marked and unmarked states, the two tasks can be exchanged also. Namely here we are searching the unmarked states. Compared to QAA [25], our algorithm adopts a different strategy in achieving $100 \%$ successful rate, we use the same phase angle in our steps, whereas the QAA, the standard angle is used in all but the last steps, and at the final step different phases are used so that the final state is the wanted state [25].

This work was supported by the Fundamental Research Funds for the Central Universities.

1 Deutsch D, Josza R. Rapid solution of problems by quantum computation. Proc Royal Soc London A, 1992, 439: 553-558

2 Shor P. Algorithms for quantum computation: Discrete logarithms and factoring. In: Proceedings of the 35th Annual Symposium on the Foundations of Computer Science, 1994. 124-134 
3 Grover L K. A fast quantum mechanical algorithm for database search. In: Proceedings of the Symposium on the Foundations of Computer Science, 1996. 212-219

4 Bennett C H, Bernstein E, Vazirani U, et al. Strengths and weakesses of quantum computing. SIAM J Comput, 1997, 26: 1510-1523

5 Simon D. On the power of quantum computation. In: Proceedings of 35th Annual Symposium on Foundations of Computer Science, 1994. 116-123

6 Fu X Q, Bao W S, Zhou C, et al. t-Bit semiclassical quantum Fourier transform. Chin Sci Bull, 2012, 57: 119-124

7 Wang X, Bao W S, Fu X Q. A quantum algorithm for searching a target solution of fixed weight. Chin Sci Bull, 2011, 56: 484-488

8 Fu X Q, Bao W S, Zhou C. Speeding up implementation for Shor's factorization quantum algorithm. Chin Sci Bull, 2010, 55: 3648-3653

9 Zhong P C, Bao W S. Quantum mechanical meet-in-the-middle search algorithm for triple-DES. Chin Sci Bull, 2010, 55: 321-325

10 Hao L, Li J L, Long G L. Eavesdropping in a quantum secret sharing protocol based on Grover algorithm and its solution. Sci China Phys Mech Astron, 2010, 53: 491-495

11 Hao L, Liu D, Long G L. An N/4 fixed-point duality quantum search algorithm. Sci China Phys Mech Astron, 2010, 53: 1765-1768

12 Hao L, Long G L. Experimental implementation of a fixed-point duality quantum search algorithm in the nuclear magnetic resonance quantum system. Sci China Phys Mech Astron, 2011, 54: 936-941

13 Page L, Brin S, Motwani R, et al. The pagerank citation ranking: Bringing order to the web. Technical Report. Stanford Digital Libraries, 1998

14 Brin S, Page L. The anatomy of a large-scale hypertextual Web search engine. Comput Networks ISDN Syst, 1998, 30: 107-117
15 Freadman M L, Tarjan R E. Fibonacci heaps and their uses in improved network optimization algorithms. J Assoc Comput Mach, 1987, 34: 596-615

16 Abuaiadh D, Kingston J H. Are Fibonacci heaps optimal? In: Proceedings of Algorithms and Computation 5th International Symposium, 1994. 442-450

17 Liu Y, Long G L. Deleting a marked basis-state from an even superposition of all basis-states with a single query. Int J Quant Inform, 2009, 7: $567-572$

18 Long G L, Sun Y. Efficient scheme for initializing a quantum register with an arbitrary superposed state. Phys Rev A, 2001, 64: 014303

19 Long G L, Zhang W L, Li Y S, et al. Arbitrary phase rotation of the marked state can not be used for Grover's quantum search algorithm. Commun Theor Phys, 1999, 32: 335-338

20 Long G L, Li Y S, Zhang W L, et al. Phase matching in quantum searching. Phys Lett A, 1999, 262: 27-34

21 Long G L, Xiao L, Sun Y. Phase matching condition for quantum search with a generalized quantum database. Phys Lett A, 2002, 294: 143152

22 Long G L. Grover algorithm with zero theoretical rate. Phys Rev A, 2001, 64: 022301

23 Long G L, Tu C C, Li Y S, et al. An $S O$ (3) picture for quantum searching. J Phys A, 2001, 34: 861-866

24 Long G L, Liu Y. Search an unsorted database with quantum mechanics. Front Comput Sci China, 2007, 1: 247-271

25 Boyer M, Brassard G, Hoyer P, et al. Tight bounds on quantum searching. In: Proceedings of the 4th Workshop on Physics and Computation, 1996. 36-43

Open Access This article is distributed under the terms of the Creative Commons Attribution License which permits any use, distribution, and reproduction in any medium, provided the original author(s) and source are credited. 